\title{
QoS Analysis of Kinematic Effects for Bluetooth HC-05 and NRF24L01 Communication Modules on WBAN System
}

\author{
Mahar Faiqurahman ${ }^{\star 1}$, Diyan Anggraini Novitasari ${ }^{2}$, Zamah Sari ${ }^{3}$ \\ 1,2,3 Universitas Muhammadiyah Malang \\ mahar@umm.ac.id¹, diyananggraini13@gmail.com², zamahsari@umm.ac.id ${ }^{3}$
}

\begin{abstract}
Wireless Body Area Network (WBAN) consists of a number of sensor nodes that are attached to the human body, and intended for monitor the human body condition. The WBAN system has several wireless communication modules that are used for sending or exchanging data between sensor nodes and gateway nodes or gateway nodes. There are some factors that are used to decide which communication modules should be implemented on WBAN system, including communication efficiency, distance range, power consumption, and the effect of mobility on QoS. In this study, we analyze the impact of the kinematic movement of sensor nodes on QoS parameter of HC-05 Bluetooth and NRF25L01 communication modules, during sending and receiving process among nodes. We assume that the sensor node and gateway node are attached on the limbs to catch the movement. We use Quality of Service (QoS) parameters such as delay, jitter, and packet loss, to analyze the impact of movement on communication modules. Based on the experimental result, it was found that the average value of delay and jitter for booth communication modules was slightly influenced by the speed of the sensor node movement. During the sensor node movement and data transmission, we found that the NRF24L01 module have a lower delay and jitter value than Bluetooth $\mathrm{HC}-05$ module. The percentage of packet loss tends to be stable at $0 \%$ value, even though the speed value becomes higher.
\end{abstract}

Keywords: Quality of Service, Bluetooth HC-05, NRF24L01, Wireless Body Area Network, Kinematic

\section{Introduction}

The Wireless Body Area Network (WBAN) system is built from a number of devices that have sensor modules, called sensor nodes, that are placed on certain body parts, and used to extract information about the condition of the human body [1]. There are several main components of WBAN architecture, namely: sensor nodes, gateway node, and data processing servers [2][3]. The sensor node is attached to the certain body part of the user and is used to retrieve data related to the activity and condition of the human body. The Gateway node collects data from various existing sensor nodes and sends those data to the server. While the server responsible for gathering and processing the data, sent by the gateway node. As well as sensor nodes, the gateway nodes are portable, small and easily carried by users.

Data, which is collected from sensor nodes, are processed by servers for various types of applications. There are some of WBAN applications that have been implemented, include healthcare applications [4][5], such as monitoring application for oxygen levels in the blood, monitoring applications for electrocardiogram (ECG) [6][7], monitoring applications for blood pressure [8], etc. This healthcare application can be integrated with mobile cloud computing to reach higher scalability [9]. In military, WBAN application can also be implemented to assess soldier fatigue and battle readiness [10]. The fall detection system is another kind of WBAN application that gather and analyze data of the body movement to detect the falling condition of elderly people [11]. The fall detection application utilizes an accelerometer and gyroscope sensor which is used to detect the sudden changes in body position. The sensors have proven to be effective in obtaining kinematic change data of the human body [12]. In another study, it was explained that the implementation of WBAN can also be applied to non-medical applications [13], including the body gesture detection system for controlling multimedia applications, emotional detection systems, authentication systems through gestures, and others.

Communication modules play an important role in the WBAN architecture. It connects the sensor node and gateway node through short distance wireless communication. Because of the

Faiqurahman, M., Novitasari, D., \& Sari, Z. (2019). QoS Analysis of Kinematic Effects for Bluetooth HC-05 and NRF24L01 Communication Modules on WBAN System. Kinetik: Game Technology, Information System, Computer Network, Computing, Electronics, and Control, 4(2). doi:http://dx.doi.org/10.22219/kinetik.v4i2.826

Receive April 24, 2019; Revise May 01, 2019; Accepted May 07, 2019 
nature of sensor nodes and gateway nodes, which have limited power resources, the communication module must be energy efficient. Only a small data rate is needed for the data transmission process between sensor nodes and the gateway node. There are a number of communication modules that are widely implemented in WBAN such as Zigbee, Bluetooth, nRF24, etc. [5]. In the previous research, the Zigbee module was used to transmit moment inertia data in the WBAN system, which is used for gait analysis [14]. From the experimental result, the Zigbee module can properly send data within a range of 4 meters. While the power consumption used was quite small. In another study [15], the author compared nRF24, XBee Pro, and Bluetooth Low Energy (BLE) communication module, which was implemented in The Blind Cane, a guide system for blind people. The author uses the signal strength in loss or no loss area condition as comparison parameters. The result shows that nRF24 and Bluetooth modules are more suitable for short distance communication.

In addition to signal strength, QoS parameters can be used to determine the quality of a wireless transmission module. There are several factors that can affect the Quality of Service (QoS) of data transmission using the wireless communication module. Not only depends on environmental conditions and distances between nodes but also influenced by the mobility of the nodes or kinematic [13][16][17]. Related to the mobility of sensor node, some of studies in area of WBAN applications are focus on creating application that utilize the data of object movement, such as in the fall detection application [11], kinematic measurement application [12], the gait analysis application [14], video game controller [18], and monitoring application for elderly people [19]. However in this previous study, authors did not analyze the influence of sensor node mobility on the quality of wireless transmission.

Based on the background described, in this paper, we analyze the effect of kinematics on Quality of Service (QoS) on short-range wireless communication modules using Bluetooth HC-05 and NRF24L01. Both modules are applied in the WBAN system for gait analysis applications. The data of movement (kinematics) from the gait analysis application was obtained from a Gyroscope sensor which was installed on the sensor node. In the last part, we made a suggestion, which communication module is best used by the WBAN application, especially the applications that are much influenced by mobility of sensor nodes.

\section{Research Method}

This study analyzed the effect of kinematics on Quality of Service (QoS) on communication protocols using Bluetooth and NRF24L01 modules, in a Wireless Body Area Network (WBAN) system. The WBAN system analyzed is used to monitor health conditions or human activities through body movements [15][20].

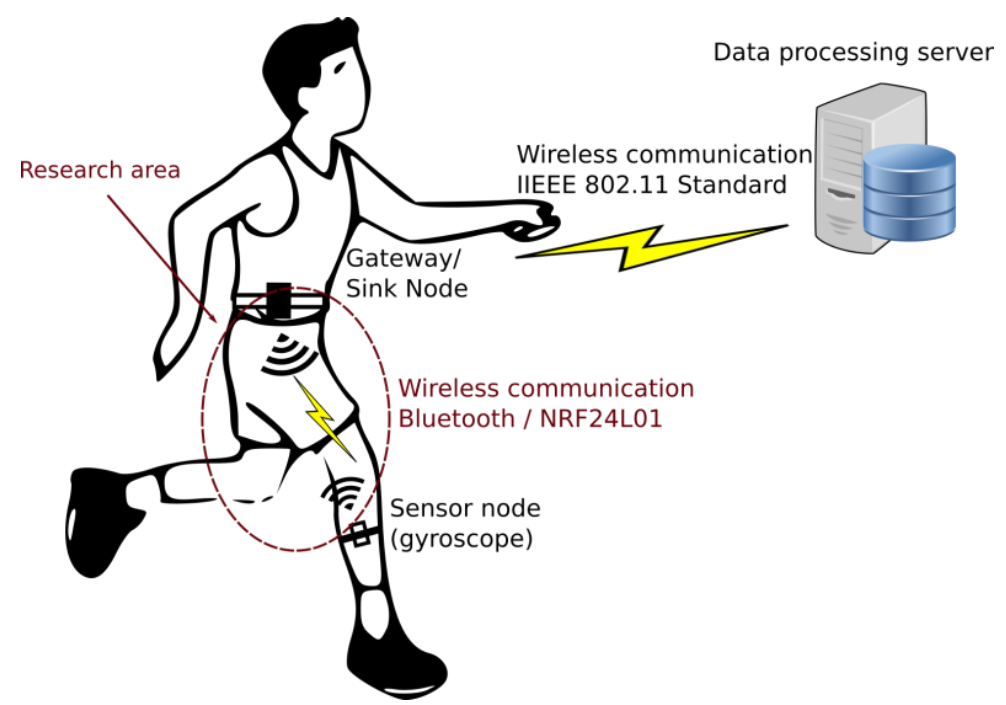

Figure 1. Sytem Architecture

The system architecture provided in Figure 1. This system consists of a sensor node, which captures the movement of human feet when walking or running. The sensor node implemented using Arduino Uno microcontroller module, which is attached the MPU6050 sensor (Gyroscope

KINETIK Vol. 4, No. 2, May 2019: 187-196 
sensor) to retrieve information related to feet movement. The sensor node is placed on the leg calf. For every movement of the feet, the Gyroscope sensor attached to the sensor node, record the movement of feet. Then, all information related to feet movement sent to Gateway. Afterward, gateway forwards the feet movement data to the data processing server. In this study we assume that we only collect data of the feet movement into gateway node for QoS analysis purpose.

The Gateway node (sink node) is another component of WBAN. Gateway node implemented using Arduino Uno microcontroller module. It collects data from sensor nodes attached on the human body, then forwards the data to the server to be further processed in the monitoring system. In this study, data which have been collected was generated as the results of the movement speed calculation carried out by the sensor node installed on the calf. This information of movement speed will be used to test and analyze the QoS of the protocols and communication modules, that influenced by the kinematic movement.

Booth sensor node and gateway node connected wirelessly using a short-range communication module, attached to Arduino Uno board. The reason why we choose the wireless communication was that the foot which was attached a sensor node can be moved freely without any disturbance from the wired communication medium. We assume that there are two types of communication modules were used, Bluetooth and NRF24L01, where the module provides a short-range communication system, with low power requirements. During the sending process, the protocol used in each communication module is adjusted to the standard protocol on Bluetooth HC-05 and NRF24I01.

\subsection{Sensing and Sending Data Scenario}

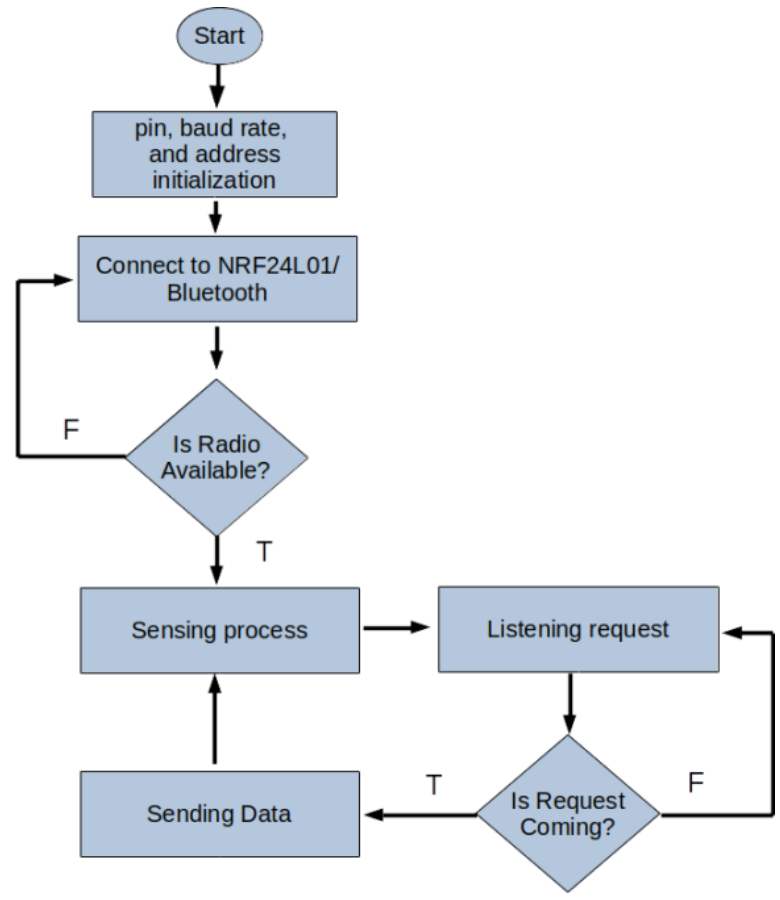

Figure 2 Sensing and Sending Data Process

In Figure 2, it can be seen that the sensing and sending data process begins with initialization of Arduino pin, baud rate, and the status of the module used, so that all of the modules are able to operate properly. After the sensor node has been activated, the sensor node will connect to the gateway node using a Bluetooth module or NRF25L01, which is attached to booth devices. If the connection failed, the sensor node will try to reconnect again. Whereas if connection was successful, the sensor module will start sensing process using Gyroscope sensor and retrieve information about the feet movement. After the results of the sensing process using gyroscope sensor are obtained, the sensor node will listen to the data request from the gateway node. If the sensor node receives a request message from the gateway node, then the sensor node will send the data to the gateway node as a response message. 


\subsection{Request-response Scenario}

As we can see in Figure 3, the process of sending requests and receiving a response of sensed data using the Bluetooth HC-05 and NRF24L01 modules also starts from the Initialization of Arduino pin, status, baud rate, and SD Card chip. For the NRF24L01 module there is an additional process, namely, address or channel initialization. In this process, the two modules installed in the sensor node and gateway nodes are connected. After the initialization, the process continues by forming a connection between the sensor node and the gateway node. After the connection has been initialized, the gateway node can send the data requested to the sensor node. If the request process does not get a response until the timeout limit, then the gateway node will resend the request message until the request process gets a response from the sensor node.

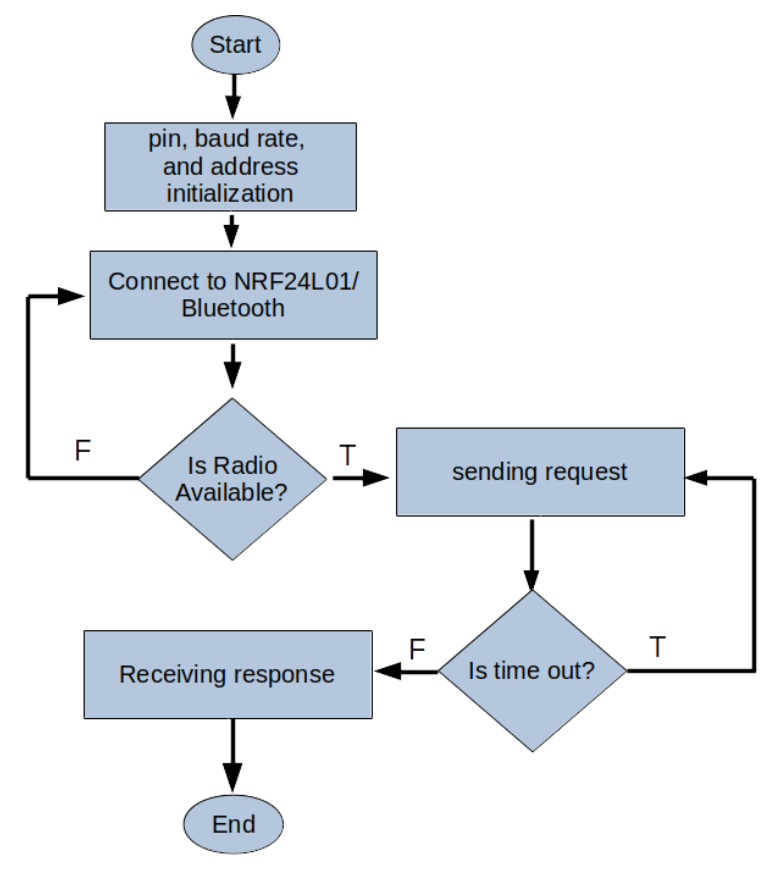

Figure 3. Sending request and receiving response on gateway node

Response data transmitted by the sensor node contains information about the changes of feet position recorded by the Gyroscope sensor. Moreover, the timestamp for each changed position of feet movement also retrieved. That information is used to calculate the speed of movement, to analyze the impact of sensor node movements on QoS of the communication modules.

\subsection{Testing Scenario}

We conducted some experiments to examine the kinematic effect toward QoS parameters on the communication module used by the sensor node. The values of delay, jitter, and packet loss were QoS parameters that have been analyzed. While the speed of movement, taken from angular velocity of the Gyroscope sensor (measure in radian per millisecond) when the feet position changed from one point to another. To simplify analysis variable we only use angular speed in $\mathrm{x}$-axis for a certain time. During the testing process, the sensor node is placed on the calf, while the gateway node is placed on the waist. Then, the users move from one place to another by walking and running at a certain speed.

During the feet movement, a request is sent by the Gateway node to the sensor node to obtain information on the movement speed that has been captured by the Gyroscope sensor. Then the sensor node sent the response data of the movement to the Gateway node. In each process of sending requests and receiving responses, Gateway node recorded timestamp t1 which is the time of sending the request, and timestamp t2 which is the time of receiving the response (can be seen in Figure 4). Then, from those values (t1 and t2) we calculate the delay (d) of data delivery using Equation 1. We define t2-t1 is round trip time. 


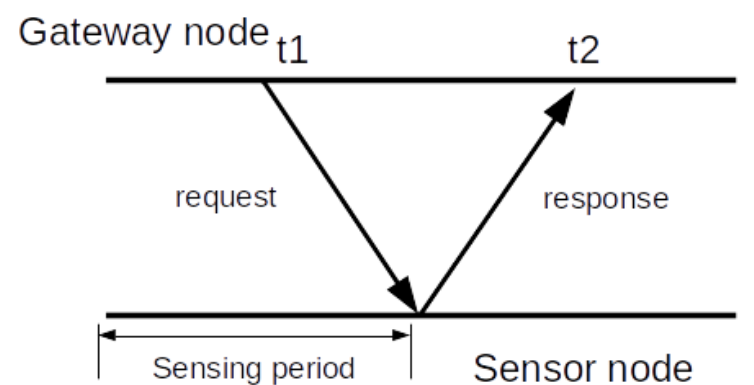

Figure 4. Request-Response Process and Recording Timestamp On Gateway Node

$$
\mathrm{d}=(\mathrm{t} 2-\mathrm{t} 1) / 2
$$

It was assumed that the sensing process on the sensor node has been done before listening and receiving the request process. So, when receiving a request, the sensor node can send the response data directly and then calculated the value of round trip time (RTT) data delivery. Another QoS parameter, jitter was obtained from average value of delay variant for a certain speed of sensor node movement. While the value of packet loss was calculated from the percentage of data lost to the data that was successfully delivered.

\section{Results and Discussion}

\subsection{The result of transmission delay for various speed of movement}

In this experiment, we made a comparison about the value of transmission delay between Bluetooth HC-05 and NRF24L01 against variations of the movement speed. The variation of speeds (Gyroscope angular speed in X-axis) is obtained from the calf movement when walking or running, while the delay transmission value was calculated from half of round trip time using equation (1). The experiment results of transmission delay provided in Table 1.

Table 1. Delay comparison between Bluetooth HC-05 anda NRF24I01

\begin{tabular}{ccc}
\hline Angular speed & \multicolumn{2}{c}{ Delay $(\mathrm{ms})$} \\
\cline { 2 - 3 } in $\mathrm{x}$-axis & Bluetooth HC-05 & NRF24I01 \\
\hline $0-50$ & 25.41 & 1.51 \\
$50-100$ & 25.41 & 1.5 \\
$100-150$ & 26.39 & 1.56 \\
$150-200$ & 26.33 & 1.56 \\
$200-250$ & 25.61 & 1.48 \\
$250-300$ & 25.97 & 0.28 \\
\hline
\end{tabular}

Comparisson of Delay Communication on Bluetooth HC-05 and NRF24101

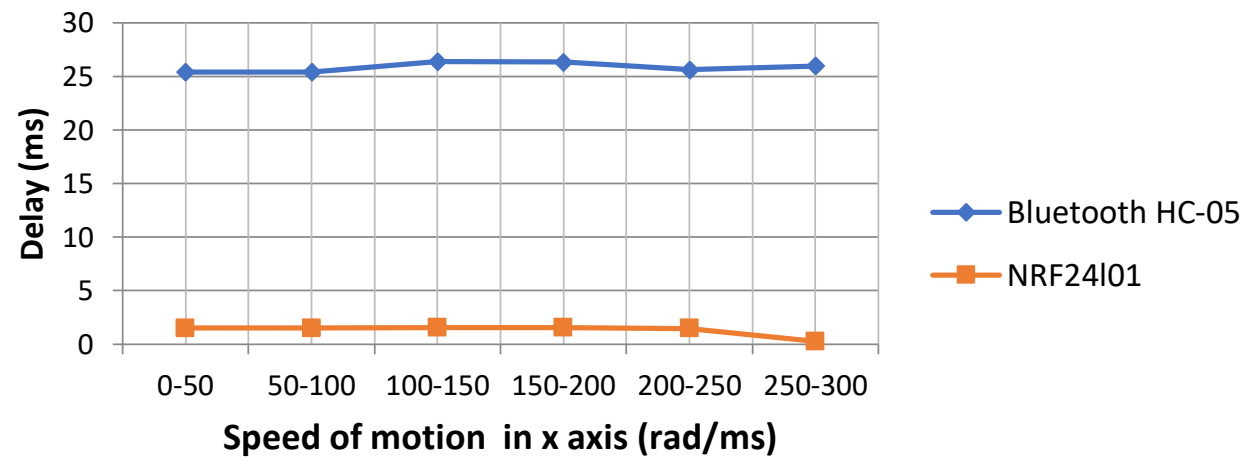

Figure 5. Delay Comparison between Bluetooth HC-05 and NRF24I01 
Table 1 and Figure 5 show the comparison of transmission delay between Bluetooth HC05 module and NRF24I01. The $y$-axis explains time delay during the data sending process (measured in millisecond), while, the $x$-axis shows the speed of sensor node movement (measured in radian/ millisecond). From the result of the experiment, we can see that Bluetooth module $\mathrm{HC}-05$ has an average transmission delay of 25.9 milliseconds. Meanwhile, the average value of the NRF24L01 transmission delay is about 1.3 millisecond. The difference in transmission delay was caused by the difference in communication protocols owned by Bluetooth $\mathrm{HC}-05$ and NRF24I01. The Bluetooth HC-05 communication protocol is more complex and has more protocol stack than NRF24I01 communication protocol. Therefore, it can increase the value of delay during data communication. Meanwhile, based on measurements in various movement speed, the value of delays have slightly fluctuated for booth modules. In low movement speed, the movement of the sensor node does not have much effect on the increase of delay value.

\subsection{The result of transmission jitter for various speed of movement}

The purpose of this experiment is to compare jitter value between Bluetooth HC-05 and NRF24L01 module during data transmission for various speed of sensor node movement. The variation of speed (Gyroscope angular speed in x-axis) also obtained from calf movement, the same as previous testing. We calculate the jitter value for every ten times of the request-response process. The results of transmission jitter testing using Bluetooth HC-05 and NRF24L01 communication module provided in Table 2.

Table 2. Jitter comparison between Bluetooth HC-05 and NRF24l01

\begin{tabular}{|c|c|c|}
\hline \multirow{2}{*}{$\begin{array}{l}\text { Angular speed } \\
\text { in } \mathrm{x} \text {-axis } \\
\text { (rad/ms) }\end{array}$} & \multicolumn{2}{|c|}{ Jitter (ms) } \\
\hline & Bluetooth HC-05 & NRF24I01 \\
\hline $0-50$ & 1.74 & 0.04 \\
\hline $50-100$ & 1.74 & 0.1 \\
\hline $100-150$ & 3.07 & 0.06 \\
\hline $150-200$ & 3 & 0.04 \\
\hline $200-250$ & 2.17 & 0.15 \\
\hline $250-300$ & 2.99 & 0.03 \\
\hline
\end{tabular}

\section{Comparisson of Jitter Communication on Bluetooth HC-05} and NRF24I01

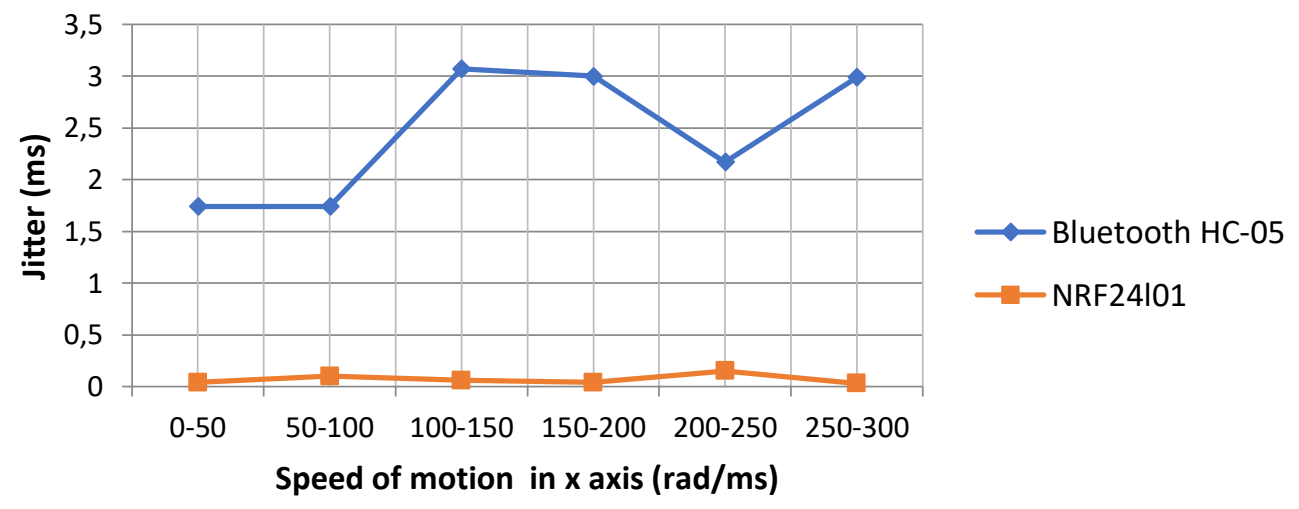

Figure 6. Jitter comparison between Bluetooth HC-05 and NRF24I01

Table 2 and Figure 6 show the jitter value of Bluetooth HC-05 and NRF24L01 modules. The $y$-axis shows the jitter value of data transmission, while the $x$-axis represents the various speed of sensor node movement. That experiment result shows that Bluetooth $\mathrm{HC}-05$ has higher jitter value than NRF24I01. The average jitter value of Bluetooth HC-05 module is 2.45 milliseconds. Meanwhile, the NRF24I01 module has an average 0.07 milliseconds. Same as the previous experiment, the difference value of jitter between two modules, is mainly due to the difference of complexity in the communication protocol. In slow movement speed (walking or

KINETIK Vol. 4, No. 2, May 2019: 187-196 
running) the increment of speed slightly influence on jitter value. The value of jitter tends to fluctuate during the experiment on booth module.

\subsection{The result of packet loss in transmission for various speed of movement}

The last experiment, we made a comparison about the value of packet loss between Bluetooth HC-05 and NRF24L01 module against variations in movement speed of sensor node. The same as the previous experiment, variation of movement speed are obtained from the user's movement while walking and running using Gyroscope sensor. The percentage of packet loss obtained from the comparison of the unsuccessfully received packet and sent a packet between the sensor node and gateway node. The comparison of packet loss during data transmission using Bluetooth HC-05 and NRF24I01 for various movement speed, provided in Table 3 and Figure 7. The $y$-axis shows the percentage of packet loss, while the $x$-axis shows the movement speed of the sensor node.

Table 3. Packet Loss Comparison between Bluetooth HC-05 and NRF24I01

\begin{tabular}{ccc}
\hline $\begin{array}{c}\text { Angular speed } \\
\text { in } x \text {-axis } \\
\text { (rad/ms) }\end{array}$ & \multicolumn{2}{c}{ Packet loss (\%) } \\
\cline { 2 - 3 } $0-50$ & 0 & NRF24I01 \\
$50-100$ & 0 & 0 \\
$100-150$ & 0 & 0 \\
$150-200$ & 0 & 0 \\
$200-250$ & 0 & 0 \\
$250-300$ & 0 & 0 \\
\hline
\end{tabular}

\section{Comparison of packet loss on Bluetooth HC-05 and NRF24I01}

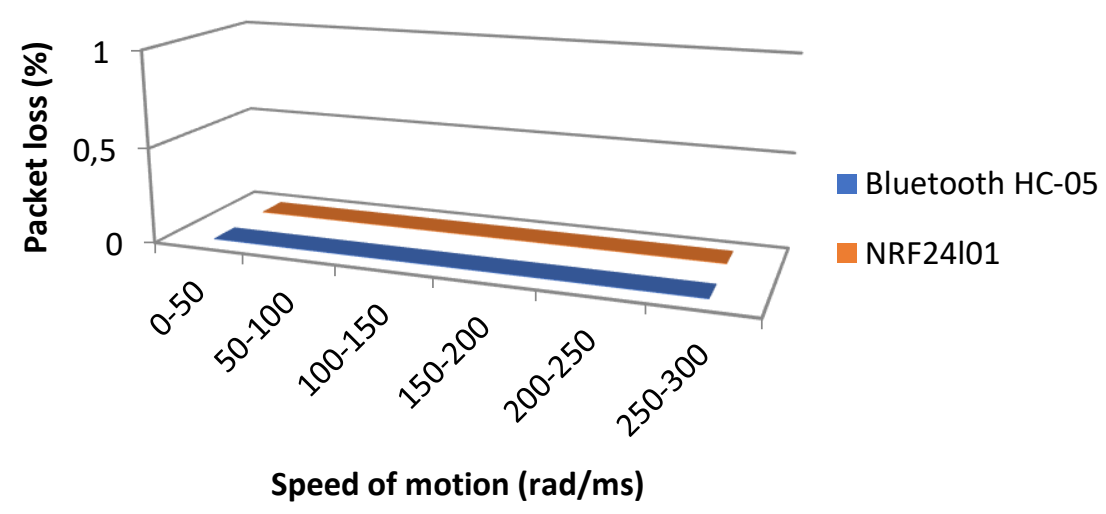

Figure 7. Packet Loss Comparison between Bluetooth HC-05 and NRF25101

Figure 7 shows that the percentage of packet loss is equal to $0 \%$ for booth modules, which means that, no missing data was found during the sending process between two nodes. The variation of movement speed, during walking or running, did not affect the change on packet loss for boot modules. This is possible due to the communication distance of the sensor node was very near to the gateway node, so it produces the good quality of signal strength.

\section{Conclusion}

In this paper, we compare and analyze the impact of kinematic on QoS for Bluetooth HC05 and NRF2101 communication modules, which were applied on the wireless body area network. Based on the experiment result, we found that the movement speed of the sensor node gives an insignificant result on delay and jitter parameters. Similarly, we found on packet loss parameter. The values of packet loss parameters for booth modules are constantly $0 \%$ for variation of movement speed. This happens because of the communication distance between two nodes is quite short, which can affect the quality of signal strength. The result also shows that Bluetooth 
HC-05 module has a higher value of delay and jitter then NRF24I01. Bluetooth HC-05 has more complex protocol rather than NRF24I01. Therefore, it can impact the increasing of computation time in sending and receiving data process, which can affect to the increment of delay and jitter value. For the overall result, we can conclude that NRF24I01 module has better performance on WBAN system rather than Bluetooth $\mathrm{HC}-05$. The module is fit to be implemented on the systems that require short distance communication.

In the future work, it is potential to investigate the influence of sensor node motion to power consumption during sending data process. This is important because the mobility of sensor node attached to human body may affect the effort of wireless communication.

\section{Notation}

t1 : time stamp when request data was sent

t2 : time stamp when response data was received

d : delay of data delivery

(t1-t2) : roundtrip time

\section{References}

[1] A. Nadeem, M. A. Hussain, O. Owais, A. Salam, S. lqbal, and K. Ahsan, "Application specific study, analysis and classification of body area wireless sensor network applications" Comput. Networks, Vol. 83, Pp. 363-380, 2015.

[2] R. Negra, I. Jemili, A. Belgith., "Wireless body area networks: Applications and technologies", Procidia Computer Science, Elsevier, 2016.

[3] M. Ghamari, B. Janko, R. Sherratt, W. Harwin, R. Piechockic, and C. Soltanpur, "A survey on wireless body area networks for ehealthcare systems in residential environments," Sensors, Vol. 16, No. 6, Pp. 831, 2016.

[4] G. V Crosby, T. Ghosh, R. Murimi, and C. A. Chin, "Wireless body area networks for healthcare: A survey," International Journal of Ad Hoc, Sensor and Ubiquitous Computing, Vol. 3, No. 3, Pp. 1, 2012.

[5] M. R. Yuce, "Implementation of wireless body area networks for healthcare systems," Sensors Actuators A Physical, Vol. 162, No. 1, Pp. 116-129, 2010.

[6] J. Liu, Y. Chen, Y. Zhou, Q. Wu, T. Qiao, and B. Sun, "Survey of Wearable EEG and ECG Acquisition Technologies for Body Area Network," in IECON 2018-44th Annual Conference of the IEEE Industrial Electronics Society, 2018, Pp. 5911-5915.

[7] A. Asif and I. A. Sumra, "Applications of Wireless Body Area Network (WBAN): A Survey," Engineering Science and Technoogy. International. Research Journal, Pp. 64-71, 2017.

[8] G. Elhayatmy, N. Dey, and A. S. Ashour, "Internet of Things based wireless body area network in healthcare," in Internet of things and big data analytics toward next-generation intelligence, Springer, Pp. 3-20, 2018.

[9] J. Wan, C. Zou, S. Ullah, C.-F. Lai, M. Zhou, and X. Wang, "Cloud-enabled wireless body area networks for pervasive healthcare," IEEE Network, Vol. 27, No. 5, Pp. 56-61, 2013.

[10] M. Salayma, A. Al-Dubai, I. Romdhani, and Y. Nasser, "Wireless Body Area Network (WBAN): A Survey on Reliability, Fault Tolerance, and Technologies Coexistence," ACM Computer Surveys, Vol. 50, No. 1, Pp. 1-38, 2017.

[11] W. Baek, D. Kim, F. Bashir, and J. Pyun, "Real life applicable fall detection system based on wireless body area network," In IEEE 10th Consumer Communications and Networking Conference (CCNC), ieeexplore.ieee.org., 2013

[12] W. A. Kusuma, Z. Sari, and A. T. Sari, "Sensor Fusion Accelerometer dan Gyroscope untuk Pengukuran Perubahan Kinematik Pergelangan Kaki," Kinetik Game Technology Information System Computer Network, Computing, Electronics, and Control, Vol. 1, No. 1, Pp. 17-22, 2018.

[13] S. Movassaghi, M. Abolhasan, J. Lipman, D. Smith, and A. Jamalipour, "Wireless body area networks: A survey,", IEEE Communications Surveys \& Tutorials ieeexplore.ieee.org, Vol. 16 , Issue: 3, 2014.

[14] M. I. Sani, "Implementasi ZigBee Transceiver untuk Akuisisi Data Sensor Inersia pada Wireless Body Area Network (WBAN)," Jurnal Infotel, Vol. 9, No. 1, Pp. 48-55, 2017.

[15] G. Mutiara, G. Hapsari, Periyadi, "Performance comparison of communication module againts detection location for blind cane," 11th International Conference on Telecommunication Systems Services and Applications (TSSA), ieeexplore.ieee.org, 2017.

KINETIK Vol. 4, No. 2, May 2019: 187-196 
[16] G. Cardone, A. Corradi, and L. Foschini, "Reliable communication for mobile MANET-WSN scenarios," in 2011 IEEE Symposium on Computers and Communications (ISCC), Pp. 1085-1091, 2011.

[17] M. Di Francesco, S. K. Das, and G. Anastasi, "Data collection in wireless sensor networks with mobile elements: A survey," ACM Transaction on Sensor Networks, Vol. 8, No. 1, Pp. 7, 2011.

[18] R. Cavallari, F. Martelli, R. Rosini, C. Buratti, and R. Verdone, "A survey on wireless body area networks: Technologies and design challenges," IEEE Communications Surveys \& Tutorials, ieeexplore.ieee.org, Vol. 16, Issue 3, 2014

[19] F. Felisberto, F. Fdez-Rivelora, and A. Pereira, "A ubiquitous and low-cost solution for movement monitoring and accident detection based on sensor fusion," Sensors, Vol. 14, No. 5, Pp. 8961-8983, 2014.

[20] M. Patel and J. Wang, "Applications, challenges, and prospective in emerging body area networking technologies," IEEE Wireless. Communication, Vol. 17, No. 1, Pp. 80-88, 2010. 
